\title{
Optical Spectra in the Ferromagnetic States near the Charge Ordering
}

\author{
S. Ishihara, and N. Nagaosa \\ Department of Applied Physics, University of Tokyo, Bunkyo-ku, Tokyo 113
}

(September 26, 2018)

\begin{abstract}
The optical conductivity is studied numerically for the ferromagnetic metallic state close to the charge ordering observed in perovskite manganites. We focus on roles of the charge fluctuation on the incoherent component in the optical spectra, and consider the Coulomb interaction in the one- and twodimensional spinless fermion models. The numerical results show that the incoherent part is not noticeable, and near the quarter-filling, most of the spectral weights are transferred to the Drude component. In the one dimensional model, there is little or no spectral weight in the energy region between the Drude weight and the remnant of the absorption edge which is pushed up with the carrier doping, in contrast to the two dimensional one. The discrepancy between our calculations and the experiments suggests that the orbital degrees of freedom play important roles.
\end{abstract}


After the discovery of High-Tc cuprates, the electronic structures in many transitionmetal oxides have been reinvestigated intensively from the renewed viewpoints. One of the attractive materials is manganese oxides with the perovskite structure $\mathrm{A}_{1-\mathrm{x}} \mathrm{B}_{\mathrm{x}} \mathrm{MnO}_{3}$ $(\mathrm{A}=\mathrm{La}, \mathrm{Pr}, \mathrm{Nd}, \mathrm{B}=\mathrm{Sr}, \mathrm{Ca})$ owing to the negative colossal magnetoresistance $(\mathrm{CMR})$ and its potential applications [1] 3]. The parent compound, $\mathrm{LaMnO}_{3}$, is an antiferromagnetic insulator [4,5], where the valence of the $\mathrm{Mn}$ ion is +3 and the electronic configuration is $\left(t_{2 g}\right)^{3}\left(e_{g}\right)^{1}$ because of the strong Hund coupling. It is noted that, as well as the spin and charge, the $e_{g}$ electron in $\mathrm{Mn}^{3+}$ ion has the orbital degrees of freedom. The layer-type antiferromagnetic spin alignment and the two-dimensional character of the spin wave [6] are attributed to the orbital ordering accompanied with the Jahn-Teller lattice distortion [7 9]. As the concentration of the divalent cation is increased, the system turns to the ferromagnetic metallic $(\mathrm{FM})$ states at $x \sim 0.175$ in $\mathrm{La}_{1-\mathrm{x}} \mathrm{Sr}_{\mathrm{x}} \mathrm{MnO}_{3}$, and $\mathrm{CMR}$ is observed near the ferromagnetic Curie temperature $\left(T_{c}\right)$. With further doping, $T_{c}$ is gradually decreased and the charge ordered (CO) phase comes out around $x \sim 0.5$ in $\mathrm{Nd}_{1-\mathrm{x}} \mathrm{Sr}_{\mathrm{x}} \mathrm{MnO}_{3}$ [10 and $\mathrm{La}_{1-\mathrm{x}} \mathrm{Ca}_{\mathrm{x}} \mathrm{MnO}_{3}$ [4]. In the $\mathrm{CO}$ phase, the spin and orbital orderings coexist. Therefore, it is recognized that the perovskite manganites are one of the typical materials where the spin, charge and orbital degrees of freedom are strongly correlated.

In the FM phase, some anomalous features in the optical conductivity $\sigma(\omega)$ are recently reported [11]. $\sigma(\omega)$ shows the two-component feature, that is, the sharp Drude peak with the small intensity and the broad incoherent band up to about $1 \mathrm{eV}$. Even at the lowest temperature, where the magnetization is almost saturated, the integrated intensity of these components is still growing up with decreasing the temperature. Because the spins are perfectly aligned at that temperature, the results indicate that another degrees of freedom are still active. In our previous paper [12], we focused on the orbital degrees of freedom in perovskite manganites and investigated the nature of the orbital fluctuation in the FM phase. With considering the strong electron-electron interactions and the anisotropy of the electron transfer, it is concluded that the long range orbital ordering is prevented by the twodimensional character of the orbital fluctuation. We proposed the orbital liquid states where 
the orbital fluctuation is still active down to low temperatures. The numerical calculation based on this scenario shows the broad spectra in the optical conductivity. It has been proposed that the orbital liquid state is one of the promising candidates for the origin of the incoherent spectra observed in perovskite manganites.

As well as the orbital degrees of freedom, the Mn ion in the FM phase has the charge degrees of freedom, as was pointed out above. In the CO phase around $x \sim 0.5$, the charge excitation gap, showing the gentle slope, is found in the optical conductivity in $\mathrm{Pr}_{0.6} \mathrm{Ca}_{0.4} \mathrm{MnO}_{3}$ [13]. Introducing the charge carriers or applying the magnetic field to the $\mathrm{CO}$ state, the charge ordering easily melts and it turns into the FM phase at low temperatures. In the FM phase near the charge ordering, the charge fluctuation is expected to be large due to the competition between the electron kinetic energy and the long range Coulomb interaction, and may bring about the incoherent component. Therefore, it is important to study to what extent the remnant of the charge ordering is relevant to the optical spectra.

In this letter, we study numerically the optical conductivity in the FM phase near the charge ordering. We focus our attention to the roles of the charge degrees of freedom, because the orbital degrees of freedom have been investigated in our previous paper [12]. We employ the exact diagonalization of the finite size systems, and conclude that its contribution is less dominant on the incoherent broad band in the region $x<0.4$ of our interest.

We consider the $e_{g}$ electron with the single orbital in the perfectly spin polarized ferromagnetic state. In order to describe the fermionic excitations in this phase, we introduce the spin- and orbital-less fermion in each Mn sites. The on-site Coulomb interactions between the $e_{g}$ electrons and the Hund coupling between the $e_{g}$ and $t_{2 g}$ spins are irrelevant, while the inter-site Coulomb interaction dominates the charge ordering and its fluctuations in this case. We consider the following Hamiltonian,

$$
H=t \sum_{<i j>}\left(c_{i}^{\dagger} c_{j}+H . c\right)+V \sum_{<i j>} n_{i} n_{j}
$$

where $c_{i}^{\dagger}$ is the spin- and orbital-less fermion operator at site $i$, and $n_{i}$ is the charge density 
operator defined as $n_{i}=c_{i}^{\dagger} c_{i}$. $t$ and $V$ is the transfer integral for the fermion and the Coulomb interaction between the nearest neighbor sites, respectively. In order to obtain the ground state wave function and the optical conductivity spectra, we utilize the exact diagonalization method i.e., the Lanczos method and the recursion method in the finite size system [14,15]. With considering the symmetry of the lattice and the capacity of the memory in the computer, the one dimensional lattice with $N_{L}=16 \times 1$ sites and the two dimensional one with $N_{L}=4 \times 4$ sites are studied. The size effects are studied in the one dimensional lattice, and the convergence of the results is confirmed in the $16 \times 1$ sites case. The periodic boundary condition is adopted to avoid the fictitious low energy excitation near the free boundaries.

In general, the optical conductivity is decomposed into the two parts, i.e., the Drude component and the regular part, as follows,

$$
\sigma(\omega)=D \delta(\omega)+\sigma^{r e g}(\omega)
$$

where $\sigma^{r e g}(\omega)$ is defined by

$$
\sigma^{r e g}(\omega)=\frac{\pi e^{2}}{N_{L}} \sum_{n \neq 0} \frac{|\langle n|j| 0\rangle|^{2}}{E_{n}-E_{0}} \delta\left(\omega-E_{n}+E_{0}\right) .
$$

In Fig. 1, we present the doping dependence of the regular part of the optical conductivity spectra in the one and two dimensional cases. The Drude part will be discussed later. In the case where the ground state is degenerate, the spectra are averaged in the momentum space. The value of $V / t$ is chosen as 12 and 4 in the one and two dimensional case, respectively, in order to true up the absorption edges. Although these values are taken to be larger in comparison with that in the actual compounds, this is for the purpose to avoid the finite size effects. The fermion concentration $n=N / N_{L}$ is identified with $x$ in manganites, and $n=0.5$ corresponds to the half-filling. In Fig. 1, the optical gap is clearly shown at $\omega \sim V$ and at $\omega \sim 3 V$ in the one and two dimensional case, respectively. This is nothing but the charge ordered insulator, where the charge alternation occurs in the one and two dimensional lattices. The optical gap corresponds to the charge transfer gap, because an 
transfer of the fermion requires the energy of $V$ and $3 V$ in the one and two dimensional cases, respectively. With decreasing the number of the fermion, the system departs from the commensurate charge ordered phase. From the results of the calculated Drude weight, it is concluded that the system is metallic, except for the half-filling case. As the system departs from $n=0.5$, in the one dimensional case (Fig. 1(a)), the energy at the absorption edge is gradually increased with the reduction of its intensity. There is little or no spectral weight between the Drude part and the remnant of the absorption edge above $V$, and the spectral weight is transferred from the later to the former with doping. This picture for the optical spectra in the one dimensional chain are consistent with results predicted by the bosonization procedure [16], where the above two components in the spectra is interpreted as the intra- and inter-band transitions at the Luther-Emery point. Near the quarter-filling $(n=0.25)$ in Fig. 1(a), the Drude part governs the almost all spectral weight and there are no remarkable weight in the finite energy region. On the other side, in the two dimensional $4 \times 4$ lattice, the charge gap is collapsed and the spectral weight appears within the gap with doping, in contrast to the one dimensional case where the remnant of the absorption edge is pushed up. This is because the charge excitations at $\omega \sim V$ and $\omega \sim 2 V$ become possible by doping. However, we cannot find any remarkable spectra in the region of $\omega<V$ in Fig. 1(b). At the quarter-filling, only the small amount of the spectra remains in the finite energy region similarly to the one dimensional case.

Next we study the doping dependence of the Drude weight. In Fig. 2, we show the Drude weight $(D)$ and the total spectral weight $(f)$, in one- and two-dimensional cases. The Drude weights in the finite size cluster with the periodic boundary condition are numerically estimated by the following relation [17, 18]:

$$
\frac{D}{e^{2}}=-\frac{\pi}{N_{L}}<T>-\frac{2}{e^{2} N_{L}} \sum_{n \neq 0} \frac{|\langle n|j| 0\rangle|^{2}}{E_{n}-E_{0}},
$$

where the first term in the r.h.s is the total spectral weight $f$, and $\langle T\rangle$ is the expectation value of the kinetic energy in the ground state. The validity of the above formula has been carefully examined in the one dimensional Hubbard model by the several authors [17, 18. 
It has been confirmed that for $N \rightarrow \infty, D$ estimated by Eq. (4) is close to the Betheansatz value, and its doping dependence is qualitative correct even in the small cluster system. At half-filling in Figs. 2(a) and (b), we find small positive and negative values of $D$ being attributed to the finite size effects. As the several authors have mentioned [17,18], it becomes inconspicuous with increasing the size and the magnitudes of the interaction. With doping into the insulating states at the half-filling, the Drude weight appears and the system becomes metallic. In the one dimensional case, $D$ is rapidly growing up with the carrier density $|0.5-n|$, and its value approaches to $f$, immediately. With further doping, both $D$ and $f$ go toward zero at $n=0.0$ and 1.0 cases. On the other hand, in the two dimensional lattice, $D$ is suppressed near the half-filling, reflecting the spectral weight in the finite frequency region, as shown in Fig. 1(b). As the system approaches to the quarterfilling case, however, almost all the weights go to the Drude component. With increasing $V / t$, the rising of $D$ and $f$ around the half-filling become slower, and the Drude part dominates the total spectra more and more.

In Fig. 3, we present the static charge correlation function $\chi_{c}(\vec{k})$ calculated in the finite size system. $\chi_{c}(\vec{k})$ is defined by $\chi_{c}(\vec{k})=F . T .<\delta n_{i} \delta n_{j}>$ with $\delta n_{i}=n_{i}-n$. At half-filling, $\chi_{c}(\vec{k})$ has a sharp peak at $\pi / a$ and $(\pi / a, \pi / a)$ in the one and two dimensional lattice, respectively, reflecting the commensurate charge ordering. With doping carriers, the intensity of $\chi_{c}(\pi / a)$ in the one dimensional lattice is drastically decreased and the broad incommensurate peak appears around $k=\pi / a-\delta$. In the cases of $n \geq 0.4375$ (more than one hole doping), the magnitude of $\chi_{c}(k)$ is smoothly reduced with the doping. On the other hand, in the two dimensional $4 \times 4$ lattice, the noticeable peak at $(\pi / a, \pi / a)$ still remains up to the case of $n=0.375$. With further doping, the intensity of the correlation function is almost saturated, and the incommensurate peak appears at $(\pi / 2 a, \pi / a)$ at the quarter filled case. The doping dependencies of $\chi_{c}(\pi / a)$ and $\chi_{c}(\pi / a, \pi / a)$ are strongly correlated with these of the Drude weight, shown in Fig. 2, both in the one and two dimensional cases, i.e., as the charge correlation at the boundary in the Brilloun zone is diminished, the Drude weight is gradually growing up. It is suggested that the strong charge correlation prevents 
the coherent motion of the doped carrier.

As we show in Figs. 1 and 2, any conspicuous spectral weight are not found in the region of $\omega<V$ in both one and two dimensional cases. This feature is quite in contrast to the results for the positive-U Hubbard and t-J models [19 21] which has been extensively studied in connection with the mid-infrared absorption observed in High-Tc cuprates. As the carriers are doped in the half-filled antiferromagnetic insulator, the spectral weight appears around $\omega \sim J \sim t^{2} / U$, in addition to the $\omega \sim U$ structure originated from the transition between the Hubbard bands in the Hubbard model. In the two dimensional case, this structure becomes remarkable and its weight is comparable to that located around $U$. The origin of this mid-infrared component is interpreted as the incoherent motion of the doped holes which creates distortions in the antiferromagnetic background. In a word, the presence of the spin degrees of freedom is essential to the emergence of the band. On the contrary, only the charge degrees of freedom is taken into account in the present model, describing the perfectly polarized ferromagnetic states without the orbital degeneracy. There are not additional degrees of freedom which govern the optical spectra smaller than $V$.

Turning to the experimental results, the frequency dependence of the conductivity spectra is measured in $\mathrm{La}_{1-\mathrm{x}} \mathrm{Sr}_{\mathrm{x}} \mathrm{MnO}_{3}$ at $\mathrm{x}=0.0$ (antiferromagntic insulator) [22], 0.1(ferromagnetic insulator), $0.175(\mathrm{FM})$ and $0.3(\mathrm{FM})$ [1]. The weight and width of the incoherent band becomes the most intense at $\mathrm{x}=0.175$ and it entirely fills up the optical gap (about $1 \mathrm{eV}$ ) observed at $\mathrm{x}=0.0$ and 0.1 . With further hole doping $(\mathrm{x}=0.3)$, the weight is rather diminished in spite that the charge fluctuation is expected to be large, as the system approaches to $\mathrm{x}=0.5$. The incoherent band also appears by applying the magnetic field in $\operatorname{Pr}_{0.6} \mathrm{Ca}_{0.4} \mathrm{MnO}_{3}$ where the charge ordering is realized without the magnetic field. [13]. At about 6.5T, the spectra are continuously spread up to about $0.8 \mathrm{eV}$ and entirely covers up the charge gap observed at zero magnetic field. On the contrary, in the present theoretical calculation, the almost all weights are transferred to the Drude part near $n=0.25$ where the charge correlation at the zone boundary are fairly weaken, and even around the half-filling, there is no noticeable spectra in $\omega<V$. Although the numerical calculations are limited in the one 
and two dimensional finite size systems, we have no doubt that the contributions from the charge fluctuation are less dominant on the incoherent component observed in the perovskite manganites.

The results presented in this paper suggest the other candidates for the origin of the broad incoherent component in the optical spectra, such as the low dimensional orbital fluctuation proposed by the present authors [12], the interband transition proposed by Shiba. et.al. [23] and so on. Actually, incoherent band based on the orbital fluctuation are calculated by the static approximation [12] where the orbital fluctuation is treated as the slowly varying degrees of freedom. It is shown that the width of the spectra is an order of the transfer integral and its weight is expected to be growing up with decreasing the temperature. Also, our previous calculation is consistent with the observed two-component feature in the optical conductivity with considering the interaction between the orbital and Jahn-Teller distortion.

\section{ACKNOWLEDGMENT}

The authors would like to thank Profs. Y.Tokura, S.Maekawa, H.Shiba, and E.Dagotto for their valuable discussions. One of the authors (S.I.) would like to express his thanks to Drs. W.Koshibae, and K.Tsutsui for their comments on the numerical calculation. This

work was supported by Priority Areas Grants from the Ministry of Education, Science and Culture of Japan, the Proposal-Based Advanced Industrial Technology R\&D Program from the New Energy and Industrial Technology Development Organization (NEDO) of Japan, and Grant-in-Aid for COE research. The part of the numerical calculation is performed in the HITACS-3800/380 supercomputing facilities in Institute for Materials Research, Tohoku University. 


\section{REFERENCES}

[1] K.Chahara et al., Appl. Phys. Lett. 62, 780 (1993).

[2] R. von Helmolt, et al.,Phys. Rev. Lett. 71, 2331 (1993).

[3] Y.Tokura, et al., Jour. Phys. Soc. Jpn. 63, 3931 (1994).

[4] E.O.Wollan, and W.C.Koehler, Phys. Rev. 100, 545 (1955).

[5] G.Matsumoto, Jour. Phys. Soc. Jpn., 29, 606 (1970).

[6] K.Hirota, et al., Jour. Phys. Soc. Jpn. 65, 3736 (1996).

[7] Goodenough, in Progress in Solid State Chemistry, Vol. 5, edited by H.Reiss, (Pergamon, London, 1971).

[8] J.Kanamori, Jour. Phys. Chem. Solids, 10, 87 (1959).

[9] S.Ishihara, et al., Physica C 263, 130 (1996), ibid. Phys. Rev. B (to be published).

[10] H.Kuwahara, et al., Science, 270, 961 (1995).

[11] Y.Okimoto, et al., Phys. Rev. Lett. 57, 109 (1995), ibid. Phys. Rev. B 55, 4206 (1997).

[12] S.Ishihara, M.Yamanaka, N.Nagaosa, (cond-mat/9606160).

[13] Y.Tokura, Y.Okimoto, T.Ishikawa, and E.Saitoh, (private communication).

[14] E.R.Gagliano, et al., Phys. Rev. Lett 26, 2999 (1987).

[15] T.Nishino, Ph.D Thesis, Osaka Univ. (1991).

[16] M.Mori, et al., Jour. Phys. Soc. Jpn. 63, 1639 (1994).

[17] J.Wagner, et al., Phys. Rev. B43, 10517 (1991), R.M.Fye, et al., Phys. Rev. B44, 6909 (1991).

[18] E.Dagotto, et al., Phys. Rev. B45, 10107 (1992). 
[19] W.Stephan, et al., Phys. Rev. B42, 8736 (1990).

[20] A.Moreo, et al., Phys. Rev. B42, 4786 (1990).

[21] R.M.Fye, et al., Phys. Rev. B45, 7311 (1992).

[22] T.Arima, et al., Jour. Phys. Soc. Jpn. 64, 2488 (1995).

[23] H.Shiba, R.Shiina, and A.Takahashi, (unpublished). 


\section{Figure captions}

Fig. 1: The optical conductivity spectra in the one dimensional 16 sites (Fig. (a)) and the two dimensional $4 \times 4$ sites (Fig. (b)). $V / t$ is chosen as $V / t=12$ and $V / t=4$ in the one and two dimensional case, respectively. The calculations are performed at $\omega+i \delta$ with $\delta / t=0.0002$

Fig. 2: The Drude weight $\left(D / e^{2}\right)$ and the total spectral weights $(\pi<T>/ N)$ in the one dimensional 16 sites (Fig. 2(a)) and the two dimensional $4 \times 4$ sites (Fig. 2(b)).

Fig. 3: The charge correlation function $\chi_{c}(\vec{k})=F . T .<\delta n_{i} \delta n_{j}>$ in the one dimensional 16 sites (Fig. 3(a)) and the two dimensional $4 \times 4$ sites (Fig. 3(b)). The parameter is chosen as $V / t=4$ in the both cases. 


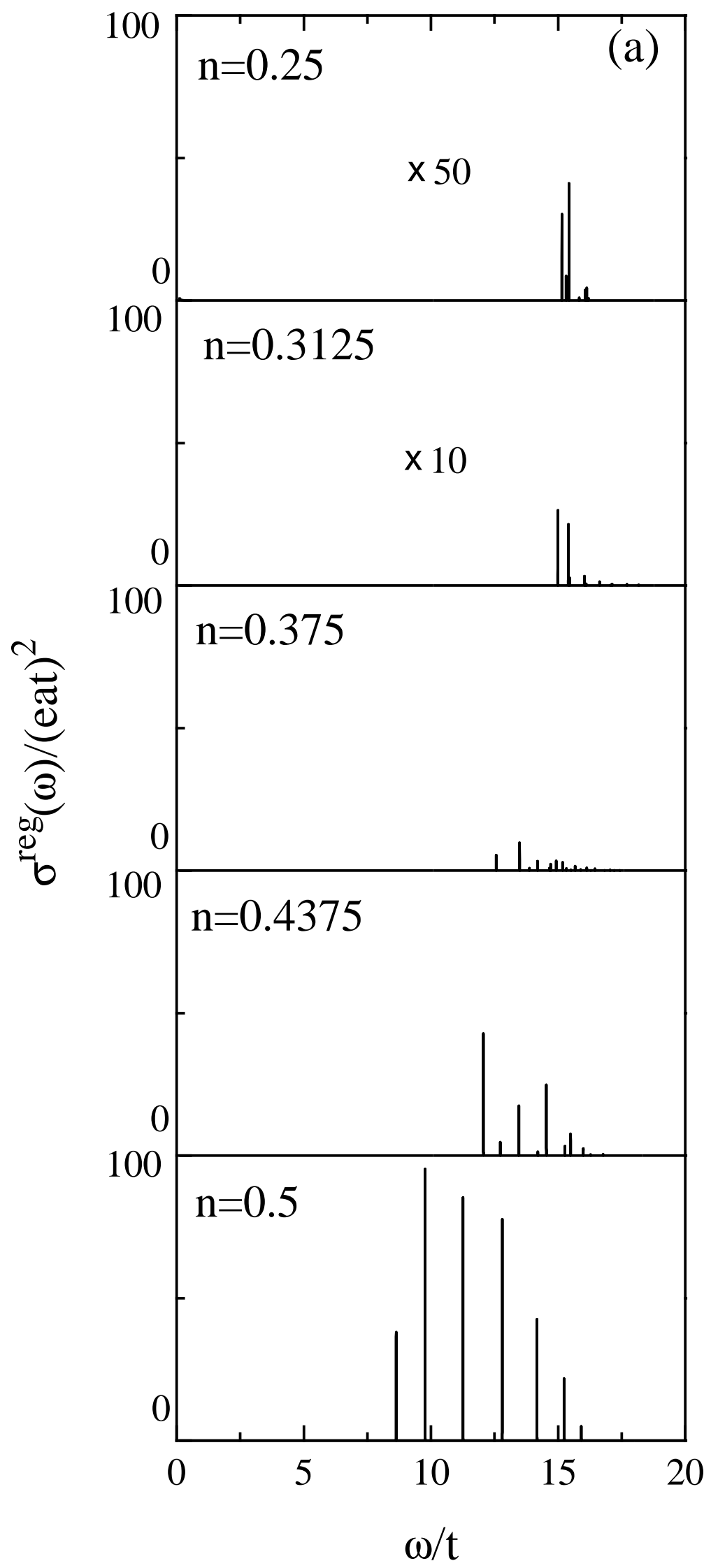




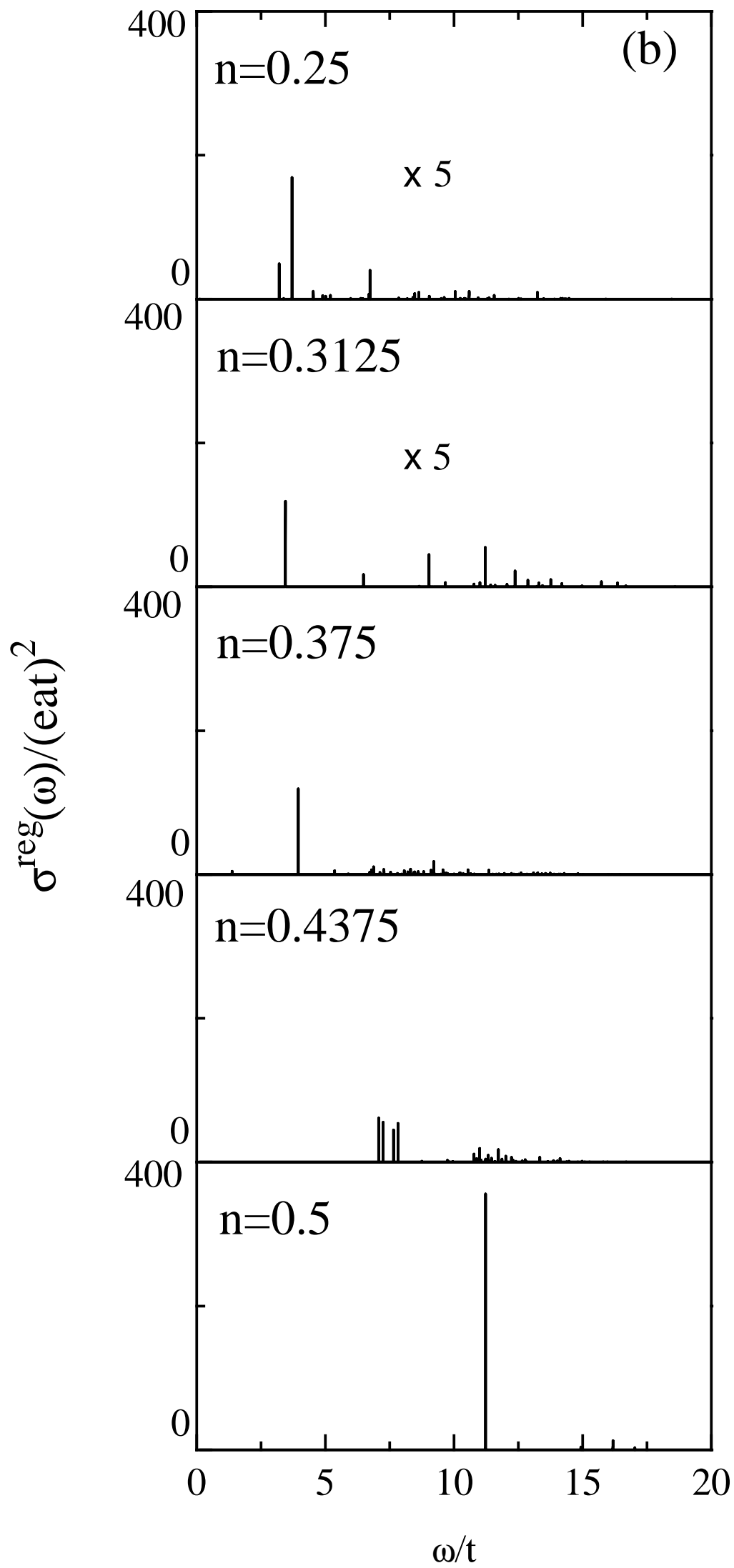




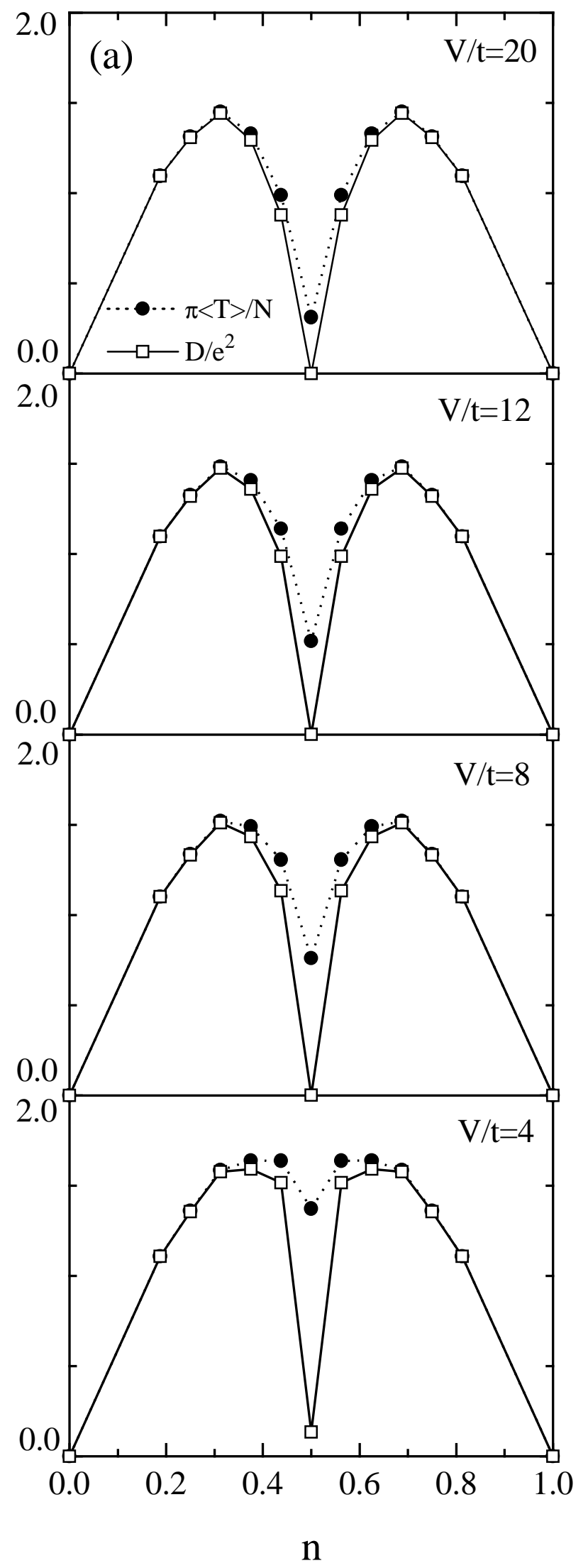




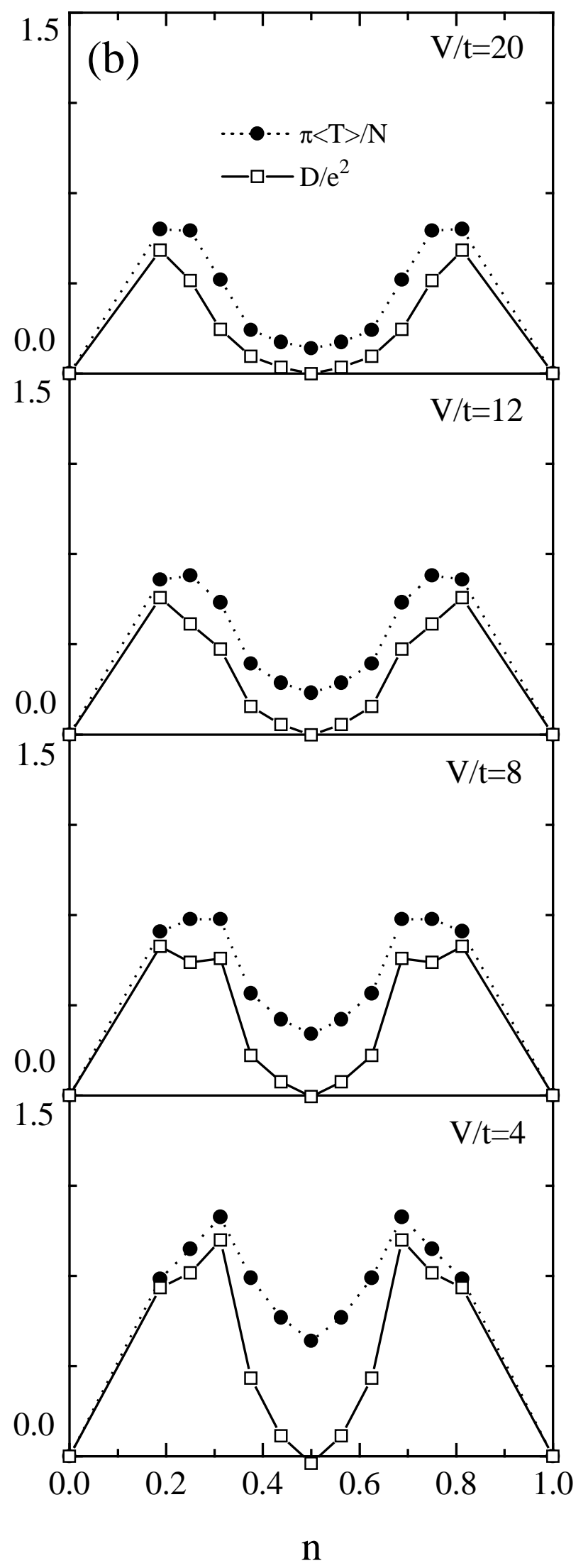




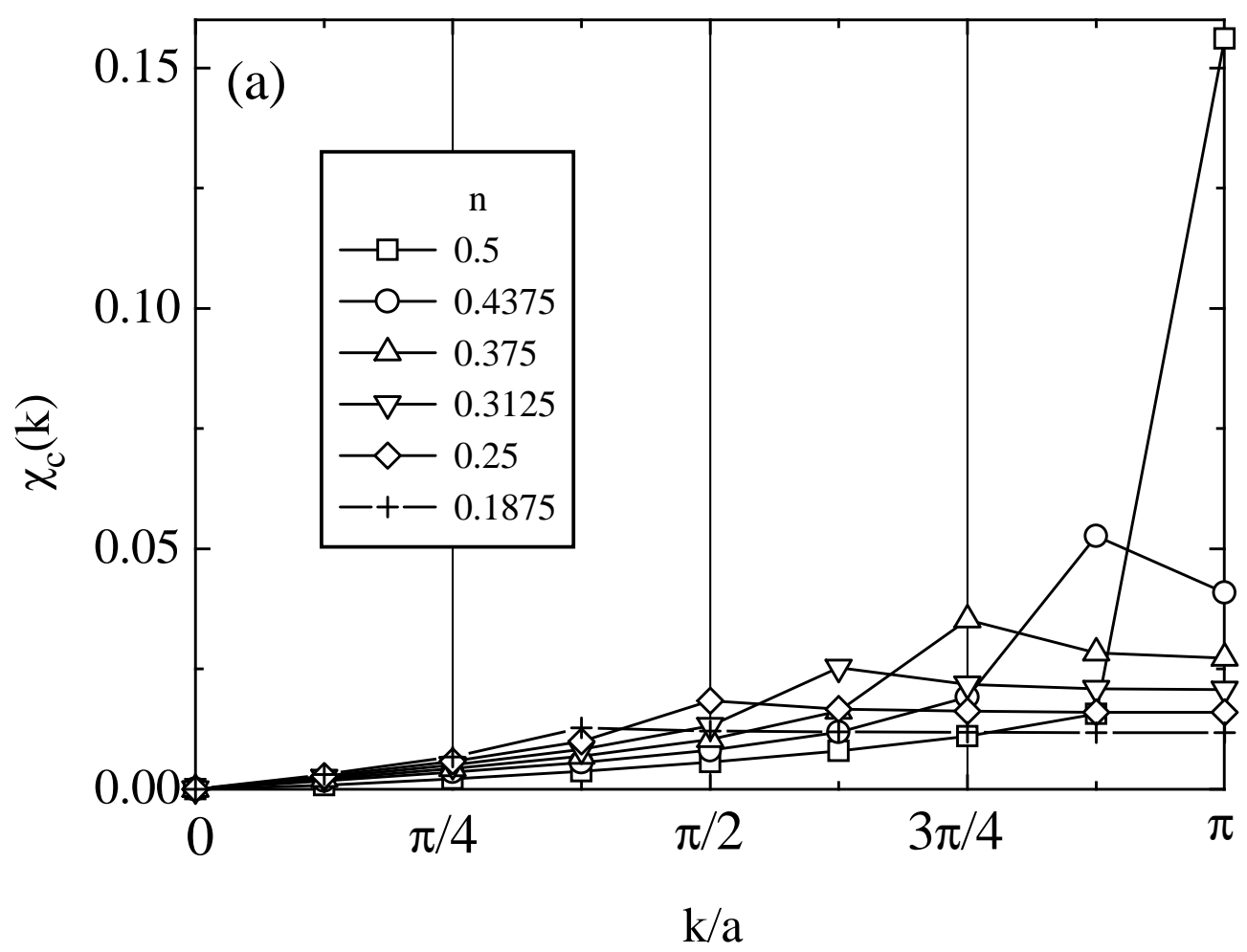




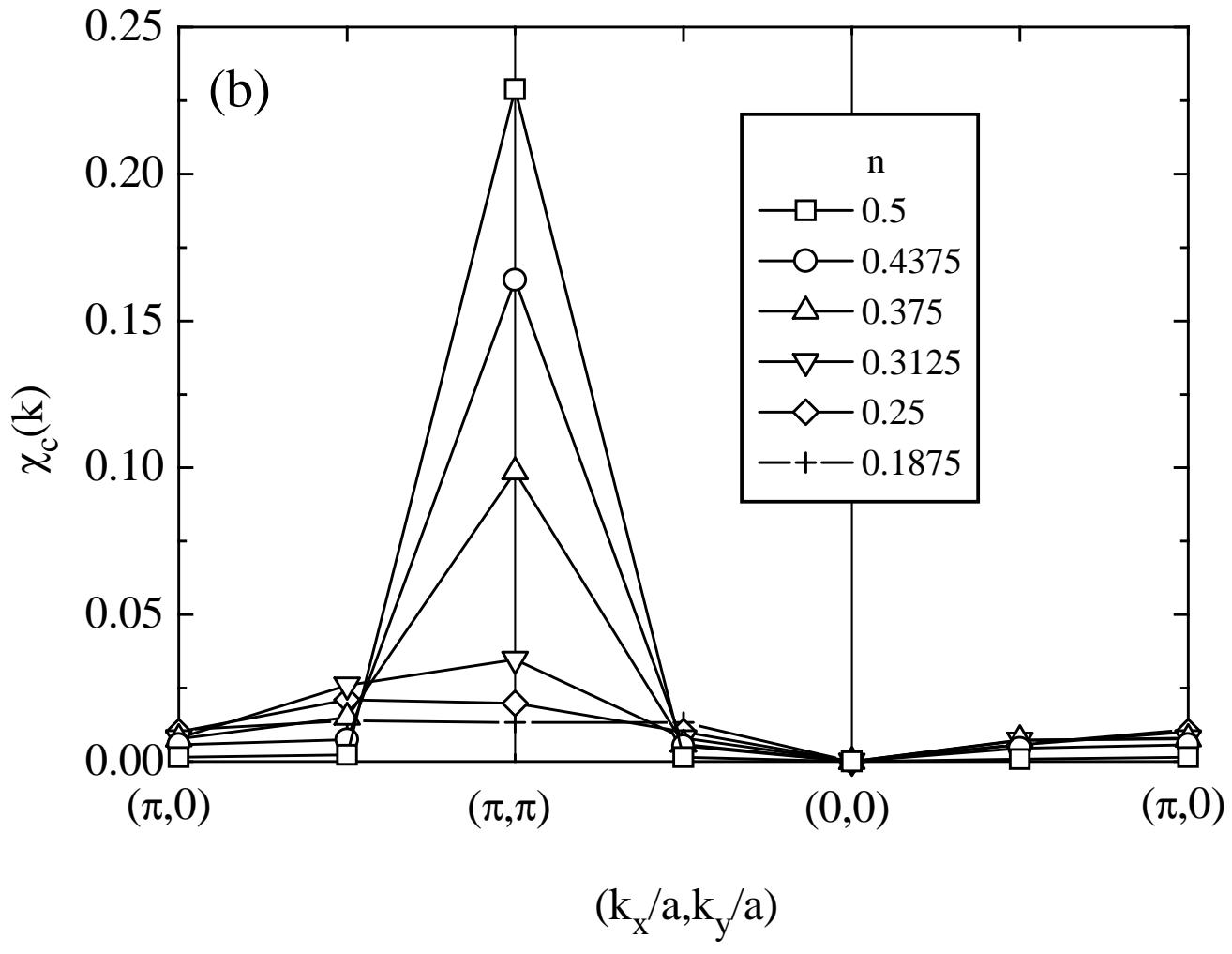

